Leading Article

\title{
The use of liposomes in the diagnosis and treatment of malignant disease
}

\author{
Isaac Levy, Jonathan Roberts and Michael Baum
}

\author{
Department of Surgery, Rayne Institute, King's College Hospital Medical and Dental School, 123 Coldharbour \\ Lane, Denmark Hill, London SE5 9NU, UK.
}

The non-specificity of pharmacologically active agents is an obstacle to their effective use either in therapeutic and diagnostic purposes or in biological research.

Recently, the ability of liposomes to encapsulate solutes, thereby sequestering them from the surrounding medium, together with the potential for sitespecific delivery in vivo has stimulated interest in their use as drugs and radio-diagnostic carriers as well. Encapsulation in the liposome also protects the drug from degradation while in transit to the target, and may alter the pharmacokinetics of distribution, metabolism and clearance.

\section{Liposomes}

When dried amphipatic lipids (water insoluble, phospholipids) are exposed to an excess of water, under the proper conditions, they spontaneously arrange themselves in extended bimolecular leaflets (concentric closed membranes) with hydrophobic chains on the interior and hydrophilic heads on the surface. Such leaflets form vesicles up to 2 microns in diameter., ${ }^{1,2}$ Upon sonication these multilamellar liposomes can break up to form smaller monolamellar structures.

Before closed structures form, there is unrestricted entry of water and solutes, (eg, drugs, enzymes) in between the planes of polar head groups. Thus, water soluble substances can be entrapped in the aqueous compartments (Figure 1). ${ }^{2}$

The carrier potential of phospholipid suspensions in medicine was predicted before (in 1935); they were described as liposomes by Bangham in $1965 .^{3}$ However, such potential became apparent in experiments, in which liposomes injected into animals were found to control both: (1) the rate of clearance of liposome-entrapped agents from the blood and (2) their destination in the body. ${ }^{4}$ The behaviour of the liposomal carrier has since been studied in vivo and in

Correspondence: Professor M. Baum Ch.M., F.R.C.S. Accepted: 30 April 1987 vitro with a number of preparations of diverse lipid composition and size, and containing a variety of substances. Several studies which have been performed in vivo showed that, while in circulation, entrapped proteins remain associated with the liposomal carrier, which imposes on them its own rate of clearance. It also appeared that there was no appreciable loss of the structural integrity of liposomes in the blood. ${ }^{5}$ Liposomes examined microscopically before and after incubation with plasma appeared to retain their spherical shape.

However in contrast to proteins, substances of low molecular weight such as penicillin $G$ and 5fluorouracil are known to diffuse out of liposomes, and such leakage can be greatly augmented in the presence of blood, possibly because of an affinity of such substances for plasma protein (albumin). ${ }^{6,7}$ On the other hand, small molecules like bleomycin or actinomycin $\mathrm{D}$ resist outward diffusion either because of their interaction with the lipid components of liposomes or because of their lipid solubility, which enables them to accommodate themselves in the lipid compartment of the carrier. ${ }^{2,8}$

The rate of removal of liposomes from the circulation is directly related to their size. With multilamellar liposomes of various size, the pattern of elimination from blood is biphasic. Removal is rapid during the first phase, but slows down during the second phase. ${ }^{9,10}$ Most circulating liposomes and their contents are taken up by tissues of the reticuloendothelial system, mainly the fixed macrophages of liver and spleen, and then by parenchymal cells of these organs. The former cells are responsible for the rapid phase of liposome clearance, whereas parenchymal cells are involved in the slow phase. Beside the liver and spleen, a variety of other organ tissues such as kidney, lungs, skeletal muscle and brain take up some of injected liposomes. ${ }^{11}$

How does this molecular 'Trojan horse' interact with cells?

It has been demonstrated that liposomes can transport 


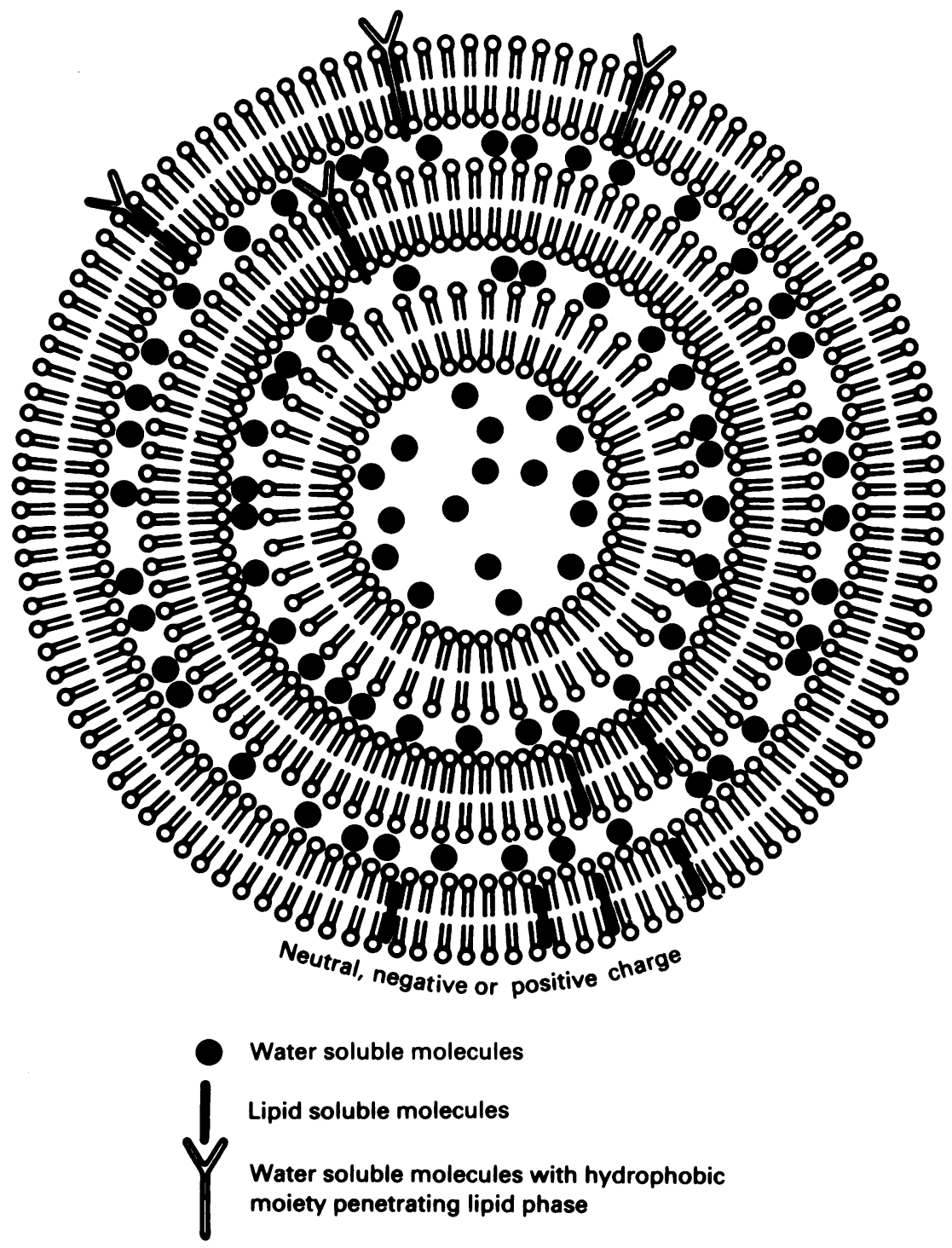

Figure 1 Schematic diagram of multilamellar liposome. These bodies can also be converted to unilamellar vesicles. (Published with permission of the author, G. Gregoriadis).

entrapped agents into cultured cells and influence intracellular catabolism. ${ }^{12}$ Liposome association with a cell is dictated to a large extent by the identity of lipids in both liposomes and cell membranes, and the homing of liposomes to the cell occurs largely through fusion of the respective membranes. After this fusion event, the aqueous contents of such vesicles are released into the cytoplasm of vesicle bound cells (Figure 2). ${ }^{13}$

The association of conventional liposomes with cells is probably a non-specific event in terms of cell $\stackrel{\sim}{\sigma}$ choice, as vividly illustrated in the living animal in $N$ which the fixed macrophages of the reticulo-endothelial system take up most of an injected dose. ${ }^{14}$ However, adjustment of the lipid composition of liposome can modify their affinity for certain types of cells. For instance, liposomes rendered positively $\stackrel{\circ}{\rightarrow}$ charged by inclusion of stearylamine in their structure are avidly bound by mouse leukaemia ${ }^{15}$ and $\mathrm{HeLa}$ cells. $^{16}$ 


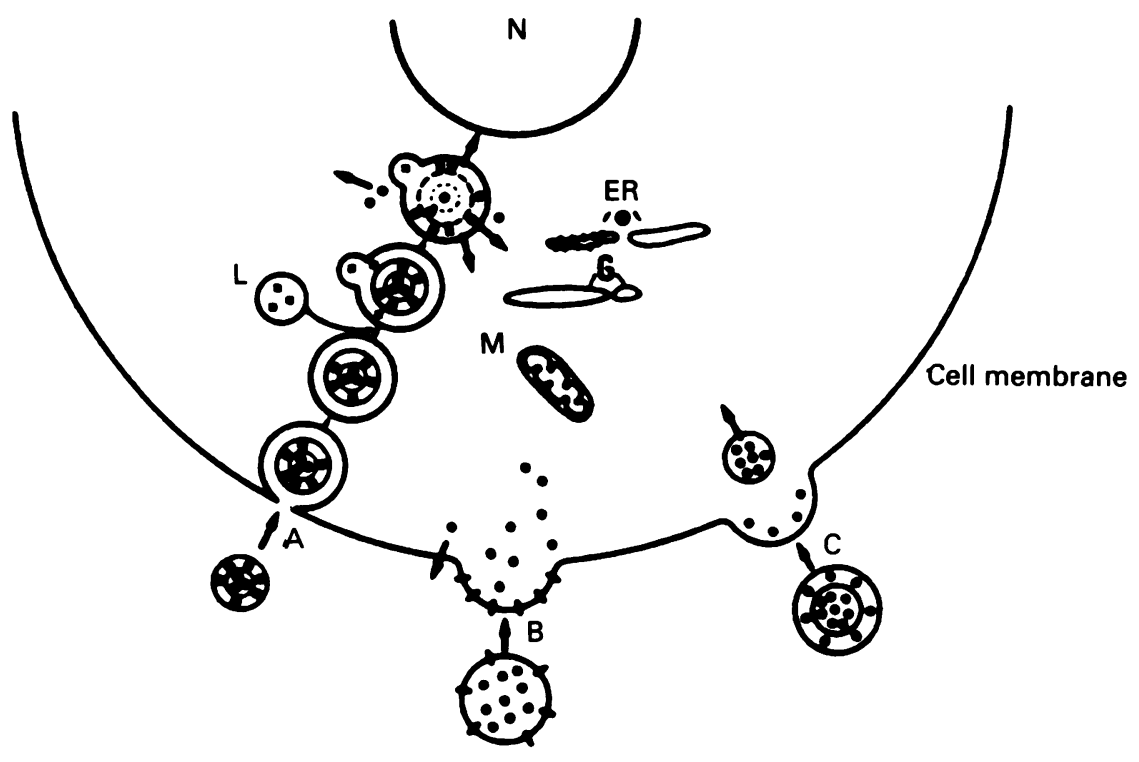

Figure 2 Possible mechanisms for cell-liposome interaction. In mechanism A endocytosis of the liposome is followed by fusion of the endocytic vacuole containing the liposome with the lysosomes. In mechanism B a unilamellar liposome fuses with the cell membrane and releases its contents to the membrane. In $\mathrm{C}$, after fusion of the multilamellar liposome, agents in the inner part of liposome and those released by fusion from the outer layer are free to interact with the intracellular contents. Both free and bound agents are able to interact with intracellular organelles; $\mathrm{N}=$ nucleus; $\mathrm{ER}=$ endoplasmic reticulum; $\mathrm{L}=$ liposome; $\mathrm{M}=$ mitochondrium.

Another approach is incorporation of cytophilic molecules onto the liposomal surface (anti-cell IgG) (homing molecule) which expedites uptake of the liposomal moiety and its contents by target cells. With IgG for instance, the Fc moiety is believed to penetrate the lipid bilayer, whereas the immunologically active Fab portions extend into the aqueous phase and become available for interactions with corresponding antigen on the surface of cells. Such antibody-targeted, drug-containing liposomes, can home to specific cell populations and in theory provide improved cancer chemotherapy by decreasing the side effects on normal cells. Recently some authors have proposed the use of liposomes conjugated to antitumour monoclonal antibodies as drug carriers for the treatment of cancer. ${ }^{17,18}$

Antibody-targeted liposomes have several potential advantages over direct antibody-drug conjugates. Many more molecules of cytotoxic agents can be delivered per molecule of antibody via liposome than with soluble antibody-drug conjugates. The targeted liposome, in order to deliver a cytotoxic drug efficiently, must bind to the target cancer cell and be internalized so that the drug can be released to the cytoplasmic compartment. Although these immunoliposomes can bind the target cell and deliver the liposomal contents to the cellular endosomes/ lysosomes, the entrapped drug can be degraded by the lysosomal enzymes and become inactive.

Recently it has been demonstrated that liposomes composed of various fatty acids such as arachidonic acid, oleic acid, palmitic acid, are fusion-competent when exposed to acidic environment. ${ }^{19}$ Exposure of liposomes of this composition to a $\mathrm{pH}$ of approximately 5.0-6.5, which is the inherent $\mathrm{pH}$ of the endosomes, will cause them to fuse with adjacent membranes and deliver the entrapped drug to the cytoplasm of the target cancer cells, while untargeted and $\mathrm{pH}$ insensitive liposomes are unable to promote cytoplasmic delivery, but only lysosomal delivery.

\section{Liposomes as carriers of radioimaging agents}

Different radioimaging agents have been used for detecting tumour, and yet none of them have been found efficient and specific. Recently many radioimaging agents composed of phospholipid vesicles containing radioisotopes have been tested for tumour imaging. Liposomes were either labelled in the lipid membrane or water soluble radiotracers were entrapped by lipid vesicles. The lipid labelling provides a more stable association of the radioactive tracer and 
the lipid vesicles, while the use of water soluble radiotracers provides a wider selection of combinations.

Early attempts at selective tumour imaging, using radio-labelled liposomes were unsuccessful. Nevertheless, gallium-67 $\left({ }^{67} \mathrm{Ga}\right)$ for instance has found widespread use as a general tumour-imaging agent. The in vivo distribution, excretion, and tumour localization of liposome-encapsulated ${ }^{67} \mathrm{Ga}$, in tumour-bearing mice have shown that ${ }^{67} \mathrm{Ga}$-labelled small unilamellar vesicles accumulate preferentially in tumours about three times higher than free ${ }^{67} \mathrm{Ga}$ NTA (nitrilotriacetic acid. $^{20}$ Technetium-99-labelled multilamellar liposomes used in patients with Hodgkin's disease have shown that the pattern of distribution of multilamellar liposomes in patients with Hodgkin's disease is different from than that of patients with other malignancies but still not adequate for the imaging of Hodgkin's disease for clinical diagnosis. ${ }^{21}$

Profitt et al. have shown increased tumour imaging potential of liposomes loaded with ${ }^{111}$ In-NTA in EMT6 tumours in BALB/C mice and concluded that small unilamellar liposomes are promising agents for the imaging of some tumours. ${ }^{22}$ Additional studies are now in progress to determine whether tumour localization of liposome entrapped radionuclides can be further improved, and different tumour types can be successfully imaged using liposomes loaded with different radioisotopes. In this domain, the use of monoclonal antibodies attached to liposomes offers new hope.

Recently improved imaging of tumour with radiotracer-labelled small unilamellar vesicles has been achieved, by using first, unloaded liposomes in order to blockade the reticuloendothelial system, on EMT6 tumour bearing mice. Biodistribution studies showed that tumours from animals with blocked reticuloendothelial systems had more than twice the

\section{References}

1. Robert, D. \& Daysun. Cell Biology: A Molecular Approach, 2nd edition. Allyn and Bacon, 1978, pp 117118.

2. Gregoriadis, G. The carrier potential of liposomes in biology and medicine. New Engl J Med 1977, 295: 704710.

3. Bangham, A.D., Hill, M.W. \& Miller, N.G.A: Preparation and use of liposomes as models of biological memranes. In Korn, E.D. (ed) Methods in Membrane Biology. Plenum Press, New York, 1974, pp 1-68.

4. Gregoriadis, G. \& Ryman, B.E. Liposomes as carriers of enzymes or drugs; a new approach to the treatment of storage diseases. Biochem J 1971, $124: 58$.

5. Gregoriadis, G. \& Ryman, B.E. Fate of protein containing liposomes injected into rats: an approach to the treatment of storage diseases. Eur J Biochem 1972, 24: 485-494. radioactivity per tissue gram than any other tissue analysed. ${ }^{23}$

There are several administration routes for liposome encapsulated imaging agents. Of particular interest is the subcutaneous administration for lymph node visualization, which can demonstrate very accurately any lymph node involvement in cancer cases. Of potential value in breast cancer, such an axillary lympho-scintigraphy might be included in the pre-operative staging of the disease and, subsequently, a better assessment of the extent of the surgical procedure.

Last but not least, liposomes may offer clear advantages over most conventional radiocontrast agents for prolonged hepatosplenic contrast enhancement. This is particularly relevant in the diagnostic evaluation of the abdominal cavity with computerized axial tomography. Important research efforts are being conducted in this field. Two different approaches have been advanced: the incorporation of contrast materials into liposomes and the preparation of radiodense lipids. Nuclear magnetic resonance imaging also may benefit by using liposomes loaded with paramagnetic elements.

\section{Conclusions}

Liposomes offer many new opportunities to vary the way in which agents are distributed through the body. Their particular biodistribution can be exploited in such diverse areas as radioimaging, cytotoxic delivery, contrast enhancement and drug administration. It is this ability to bypass many well known routes of biodistribution which offers new opportunities. As with many new technologies, development of applications will be prolonged, but is only limited by people's imagination.

6. Black, C.D.V. \& Gregoriadis, G. Interaction of liposomes with blood plasma proteins. Biochem Soc Trans 1976, 4: 253-256.

7. Gregoriadis, G. Drug entrapment in liposomes; possibilities of chemotherapy. Biochem Soc Trans 1974, 2: 117-119.

8. Dapergolas, G., Neerunjun, E.D. \& Gregoriadis, G. Penetration of target areas in the rat by liposome associated bleomycin, glucose oxidase and insulin. FEBS Lett 1976, 63: 235-239.

9. Mayhew, E. \& Papahadjoulos, D. Therapeutic applications of liposomes. In Ostro, M.J. (ed) Liposomes, Marcel Dekker, New York, 1983, pp 289-336.

10. Yatvin, M.B. Clinical prospects of liposomes. Med Phys 1982, 9: 145-175.

11. Kasi, L.P., Lopez-Berestein, G., Mehta, K. et al. Distribution and pharmacology of intravenous Tc-99m- 
labelled multilamellar liposomes in rats and mice. Int $J$ Nucl Med Biol 1984, 11: 35-37.

12. Gregoriadis, G. \& Buckland, R.A. Enzyme containing liposomes alleviate a model for storage disease. Nature 1973, 244: 170-172.

13. Pagano, R.E. \& Huang, L. Interaction of phospholipid vesicles with cultured mammalian cells. Nature 1974, 252: $166-167$.

14. Gregoriadis, G. Structural requirements for the specific uptake of macromolecules and liposomes by target tissues. In Tager, J.M., Hooghwinkel, G.J.M. \& Daems, W.Th (eds) Enzyme Replacement Therapy of Lysosomal Storage Diseases. North Holland Publishing Company, Amsterdam, 1974, pp 131-148.

15. Magee, W.E. \& Miller, O.V. Liposomes containing antiviral antibody can protect cells from virus infection. Nature 1972, 235: 339-341.

16. Magee, W.E., Goff, C.W. \& Schoknecht, J. The interaction of cationic liposomes containing entrapped horseradish peroxidase with cell in culture. J Cell Biol 1974, 63: $492-504$.

17. Gregoriadis, G. (ed) Drug Carriers in Biology and Medicine. Academic Press, New York, 1979.
18. Heath, T.D., Fraley, R.T. \& Papahadjopoulos, D. Antibody targeting of liposomes: cell specificity obtained by conjugation of $\mathrm{F}\left(\mathrm{Ab}_{2}\right)$ to vesicle surface. Science (Wash DC) 1980, 210: 539-541.

19. Connor, J. \& Huang, L. pH sensitive immunoliposomes as an efficient and target specific carrier for antitumour drugs. Cancer Research 1986, 46: 3431-3435.

20. Ogihara, I., Kojima,S. \& Jay, M. Tumor uptake of 67Ga-carrying liposomes Eur J Nucl Med 1986, 11: 405411.

21. Perez-Soler, R., Lopez-Berestein, G., Kasi, L.P. et al. Distribution of technetium-99-labelled multilamellar liposomes in patients with Hodgkin's disease. $J$ Nucl Med 1985, 26: 743-749.

22. Proffitt, T.R., Williams, L.E., Presant, C.A. et al. Tumor-imaging potential of liposomes loaded with In111-NTA: biodistribution in mice. J Nucl Med 1983, 24: 45-51.

23. Proffitt, T.R., Williams, L. E., Presant, C.A. et al. Liposomal blockade of the reticuloendothelial system: improved tumor imaging with small unilamellar vesicles. Science 1983, 220: 502-505. 